\title{
Livistona chinensis (Jacq.) R.Br. ex Mart. (Arecaceae): uma alternativa para produção de biodiesel
}

\section{Maria Gabriela da Silva Cavalcanti ${ }^{1}$, Ivan da Costa Silva ${ }^{2}$, Cláudia Teresa Teles Farias ${ }^{2}$ e Luiz Antonio Pimentel Cavalcanti ${ }^{2}, *$}

${ }^{1}$ Instituto Federal de Educação, Ciência e Tecnologia da Bahia. Curso de Engenharia Elétrica. Campus Salvador. Rua Emídio dos Santos S/N. Bairro do Barbalho. Salvador-BA, Brasil (CEP 40301-015).

${ }^{2}$ Instituto Federal de Educação. Ciência e Tecnologia da Bahia. Campus Salvador. Rua Emídio dos Santos S/№. Bairro de Barbalho. Salvador-BA, Brasil (CEP 40301-015).*E-mail: luiz.cavalcanti@ifba.edu.br.

Resumo. A avaliação de rotas para implementação e utilização de fontes de energia alternativas tem aumentado substancialmente nas últimas décadas, em virtude da redução das reservas de óleo cru e os impactos ambientais relacionados à poluição atmosférica e mudanças climáticas devido ao uso de combustíveis fósseis. Nesta perspectiva, o biodiesel surge como potencial substituto para o diesel, porém a falta de competitividade econômica ainda é um empecilho para uma maior utilização do combustível em larga escala. Alternativas de redução de custos principalmente relacionados à matéria prima são demandas emergentes para produção do biodiesel. 0 presente trabalho tem por objetivo avaliar o potencial dos frutos de Livistona chinensis (Jacq.) R.Br. ex Mart. (Arecaceae) (palmeira leque-da-China) para produção biodiesel. 0 processo de esterificação foi utilizado para prétratamento dos ácidos graxos livres (AGL) presentes na matériaprima, seguido de transesterificação alcalina para obtenção do biodiesel. As amostras de óleo da palmeira leque-da-china e seu respectivo biodiesel foram avaliados por cromatografia gasosa. A Composição do biodiesel em termos de ésteres metílicos de ácidos graxos (EMAG) foi 2,9\% (C12:0), 1,3\% (C14:0), 52,8\% (C16:0), 2,8\% (C18:0), 3,1\% (C16:1), 33,7\% (C18:1), 3,4\% (C18:2) e 0,1\% (C18:3).

Palavras-chave: Palmeira leque-da-China; Biodiesel, Bioenergia, Energia renovável.

Abstract. Livistona chinensis (Jacq.) R.Br. ex Mart. (Arecaceae): An alternative for biodiesel production. The evaluation of routes to implement and use alternative energy sources has been increased substantially at the last decades due to the reduction of petroleum reserves and the environmental
Recebido

$20 / 02 / 2020$

Aceito

$24 / 04 / 2020$

Disponível on line $25 / 04 / 2020$

Publicado

$30 / 04 / 2020$

Acesso aberto

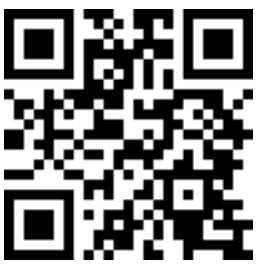

ORCID

() 0000-0002-3112-6795 Maria Gabriela da Silva Cavalcanti

0000-0001-5990-5179

Ivan da Costa Silva

ISSN 2359-1412/RBGAS-2020-0024/2020/7/15/17/233

Rev. Bras. Gest. Amb. Sustent.

http://revista.ecogestaobrasil.net 
impacts related the atmosphere pollution and climated changes all because of the use of fossil fuels. On this perspective, biodiesel appears as a potential substitute for diesel, but the noncompetition on economics ways is still an embarrassment for a greater use of fuels on large scale. Alternatives of spending reduction principally related of raw materials are emerging demand for biodiesel production. This current work has a purpose to value the potential biodiesel production of the fruits of Livistona chinensis (Jacq.) R.Br. ex Mart. (Arecaceae) (Chinese fan palm). The esterification process was used for the first treatment of free fatty acids (FFA) on raw material followed by an alkaline transesterification to acquire biodiesel. The samples of the oil of fan-of-china palm and your respective biodiesel were evaluated by gaseous chromatography. The compositon of biodiesel on methyl esters of fatty acids (MEFA) were 2.9\% (C12:0), 1.3\% (C14:0), 52.8\% (C16:0), 2.8\% (C18:0), 3.1\% (C16:1), 33.7\% (C18:1), 3.4\% (C18:2), and 0.1\% (C18:3).

Keywords: Chinese fan palm; Biodiesel; Bioenergy; Renewable energy.

\section{Introdução}

A crescente busca por combustíveis alternativos renováveis visa a reduzir a dependência da matriz energética mundial que, atualmente, atende cerca de $80 \%$ da demanda por combustíveis fósseis. Por se tratar de uma fonte não renovável de energia, a expectativa de falta de disponibilidade em um futuro próximo, associado a preocupação com a poluição atmosférica e as mudanças climáticas fazem que a comunidade científica busque alternativas para suprir as demandas energéticas do mundo moderno (Nascimento e Cavalcanti, 2017).

Neste contexto o biodiesel, surge como um substituto potencial para o diesel derivado do petróleo, podendo ser utilizado diretamente em motores de combustão ciclo diesel sem qualquer alteração mecânica nos mesmos. Com a implantação do Programa Nacional de Produção e Uso do Biodiesel (PNPB) em 2004 o percentual de mistura do biodiesel no diesel era apenas $2 \%$ (voluntário). A partir do ano de 2008, tal percentual de mistura tornou-se obrigatório. Posteriormente outros aumentos foram autorizados como, por exemplo, 7\% em 2016 e 10\% em março de 2018. Em setembro de 2019 passou a vigorar a mistura de $11 \%$ do biodiesel no diesel, também conhecida como misturas B11 (ANP, 2019).

A comunidade científica tem intensificado os estudos nas áreas de processamento e novas matérias primas com o objetivo de reduzir custos de produção. Haja vista que se projeta que o custo com matéria prima compromete cerca de até $80 \%$ do custo de produção. Nesse contexto a busca por fontes oleaginosas que apresentem alto teor de óleo, características físicas e químicas compatíveis com a reação de transesterificação alcalina e que não concorram com a produção de alimentos é uma demanda urgente do mercado atual (Verma e Sharma, 2016; Suwanno et al., 2017).

O Brasil apresenta uma ampla diversidade de palmeiras (Família Arecaceae) com notório potencial para produção de óleos e que posteriormente poderia ser aplicado para geração de agroenergia, mais especificamente a produção de biodiesel. A palmeira palmeira leque-da-China (Livistona chinensis (Jacq.) R.Br. ex Mart.) é uma espécie nativa da China, Japão e Taiwan. No Brasil é muito encontrada em parques e jardins por sua 
característica ornamental, seus frutos são ovais com coloração verde-azulados com diâmetros de 1,5 a $2 \mathrm{~cm}$ e polpa alaranjada (Costa e Marchi, 2008). Segundo Nwosu et al. (2012) a massa específica o óleo da palmeira leque-da-China é de $863,8 \mathrm{~kg} \cdot \mathrm{m}^{-3}$, que representa um valor inferior quando comparado a outras fontes oleaginosas.

Assim, o presente estudo teve por objetivo avaliar a utilização do óleo dos frutos da palmeira leque-da-China para produção de biodiesel.

\section{Materiais e métodos}

Os frutos foram coletados na região de Paulo Afonso, em dezembro de 2019. Posteriormente foram sanitizados com água clorada a $120 \mathrm{mg} \cdot \mathrm{L}^{-1}$ de cloro ativo, por 10 min e submetidos a secagem artificial com circulação de ar com temperatura na faixa de $60{ }^{\circ} \mathrm{C}$ e $70{ }^{\circ} \mathrm{C}$ por $24 \mathrm{~h}$. Após o procedimento de secagem os frutos foram descascados manualmente e acondicionados em recipiente fechado. Na Figura 1, apresenta-se a fotografia dos frutos da palmeira leque-da-China após a colheita.

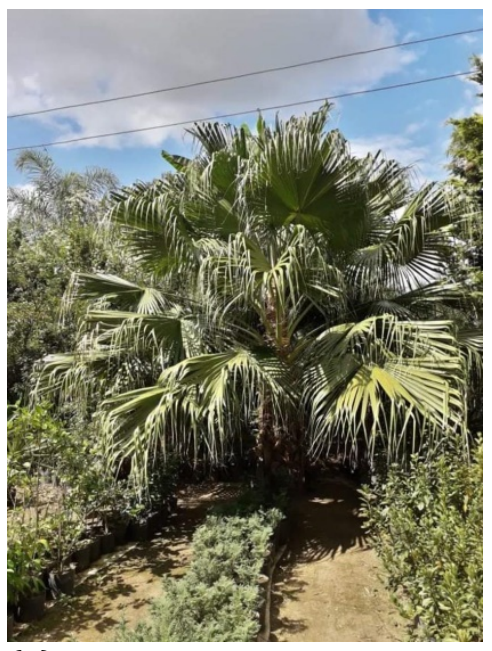

(a)

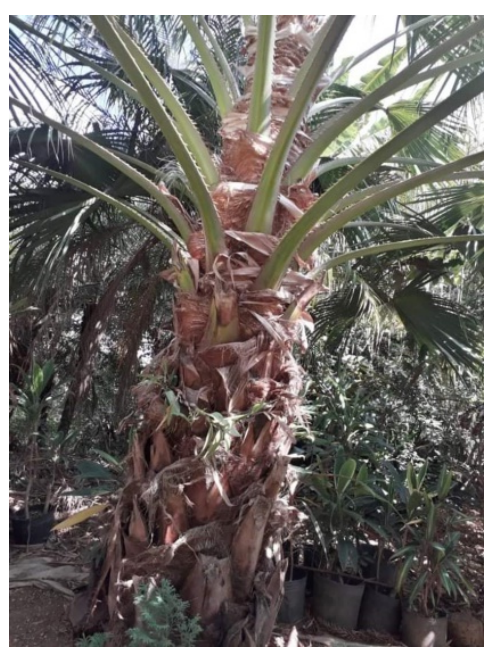

(b)

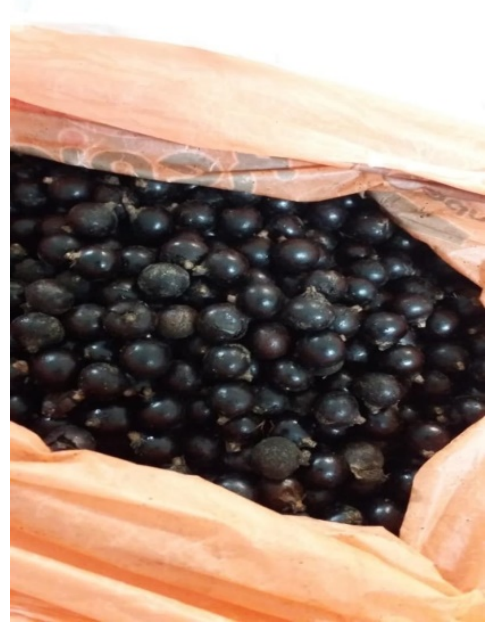

(c)

Figura 1. Palmeira leque-da-China (a) e (b); frutos após a colheita (c).

\section{Extração do óleo}

As amostras dos frutos de palmeira leque-da-China foram maceradas para aumentar a superficie de contato e colocadas em envelopes confeccionados em papel filtro. A extração por solvente foi realizada pelo método soxhlet utilizando como solvente o hexano por um período de 1 hora.

No extrator, o hexano foi aquecido até evaporar. 0 vapor de hexano passou por um condensador fazendo com que o hexano ficasse líquido novamente e fosse depositado no recipiente com a amostra. Posteriormente o hexano utilizado no processo foi retirado por destilação deixando assim a amostra de óleo isenta de solvente.

\section{Degomagem}

A degomagem do óleo bruto de palmeira leque-da-China foi realizada em um reator químico da marca Marconi, modelo MA502/5/C (volume útil máximo: 3L) com controle de temperatura e agitação mecânica (impelidor do tipo pás). Com adição 2,5\% de água e $0,1 \%$ de ácido cítrico (solução a $50 \%$ ), em relação ao peso inicial da amostra e 
agitação constante de $200 \mathrm{rpm}$ por 5 minutos à temperatura de $80^{\circ} \mathrm{C}$. Em seguida a mistura resultante foi centrifugada por 10 min para remoção da goma.

\section{Neutralização}

A etapa de neutralização foi realizada mediante a adição de 1,5\% de $\mathrm{NaOH}$ ( $\left.29^{\circ} \mathrm{Bé}\right)$, em relação a massa da amostra, a $80{ }^{\circ} \mathrm{C}$ e mantida por agitação por 3 min. Foi realizada uma reneutralização com $50 \%$ de excesso de $\mathrm{NaOH}$ nas mesmas condições anteriormente citadas para neutralização inicial.

\section{Clarificação}

Alíquotas de $50 \mathrm{~mL}$ do óleo degomado e neutralizado foram colocadas em frascos erlenmeyer de $250 \mathrm{~mL}$, os quais foram transferidos para um banho de glicerol a $90^{\circ} \mathrm{C}$. Em seguida, adicionou-se carvão ativado e a mistura resultante foi mantida sob agitação constante por 20 minutos. Transcorrido o intervalo de tempo determinado, realizou-se uma filtração à vácuo $(30 \mathrm{mmHg})$. A Figura 2 apresenta a condição final do óleo de palmeira leque-da-China após os processos de purificação.

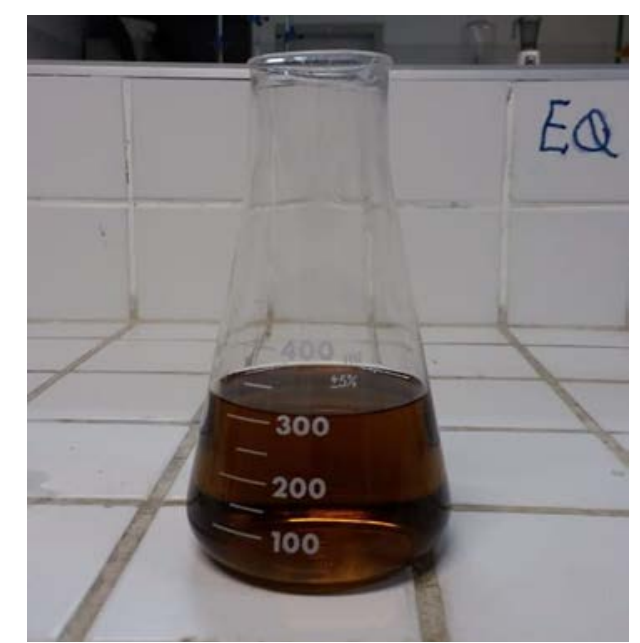

Figura 2. Óleo de palmeira leque-da-China após o processo de purificação.

\section{Determinação do índice de acidez}

A massa de $3 \mathrm{~g}$ da amostra de óleo foi medida com precisão de $0,001 \mathrm{~g}$ em balança analítica (Sartorius, modelo BL120S). Foram adicionados $25 \mathrm{~mL}$ de uma solução de éter etílico-álcool etílico (2:1) em presença de fenolftaleína (solução em etanol a $1 \% \mathrm{~m} / \mathrm{v}$ ) homogeneizando até total dissolução da amostra. 0 material foi titulado com hidróxido de sódio 0,1 mol. $\mathrm{L}^{-1}$ padronizado. Também foi realizada a titulação apenas com os solventes (branco). Ao final da titulação, foi anotado o volume de solução gasto, o qual empregou-se para o cálculo do índice de acidez (IA), conforme Equação (1):

$\% I A=\frac{V \cdot M \cdot F \cdot 282}{p} \cdot 100$

Sendo: 282 Massa Molar do Ácido Oleico; M a Molaridade da Solução Padronizada de $\mathrm{NaOH}$ (em mol.L ${ }^{-1}$ ); $\mathrm{V}$ é o volume gasto de solução titulante (em L); F é o fator de correção da solução titulante e $p$ é o número de gramas da amostra empregada no ensaio (g). 


\section{Produção do biodiesel}

O biodiesel do óleo da palmeira leque-da-China, 2L, foi produzido em duas etapas reacionais realizadas em série:

a) Esterificação ácida (catalisador: formado a partir da dissolução do $\mathrm{H}_{2} \mathrm{SO}_{4}$ em metanol), e

b) Transesterificação alcalina (catalisador: formado a partir da dissolução do KOH em metanol).

O reator químico foi de marca Marconi, modelo MA502/5/C (volume útil máximo: 3L) com controle de temperatura e agitação mecânica (impelidor do tipo pás). As condições operacionais dessas etapas foram:

a) Esterificação ácida: $60{ }^{\circ} \mathrm{C}, 1 \mathrm{~atm}, 300 \mathrm{rpm}$, razão molar álcool metílico/óleo empregada foi de 2:1, 1,0\% de $\mathrm{H}_{2} \mathrm{SO}_{4}$, em relação à massa de óleo, tempo reacional de $1 \mathrm{~h}$, e

b) Transesterificação alcalina: $60^{\circ} \mathrm{C}, 1$ atm, $300 \mathrm{rpm}$, razão molar álcool 6:1, tempo reacional de $1 \mathrm{~h}$.

Em seguida, procedeu-se a separação da glicerina da mistura reacional (método da decantação). A fase mais leve da mistura, contendo biodiesel, catalisador, excesso de metanol, passou pela etapa de purificação que constou de evaporação do metanol com uso de um evaporador rotativo a vácuo, lavagem ácida com solução de $\mathrm{HCl} 0,5 \mathrm{M}$, lavagem com água destilada e secagem em evaporador rotativo.

\section{Composição química}

A composição química do óleo e do biodiesel estudados foram obtidos por cromatografia gasosa (cromatógrafo de marca Ciola Gregory, modelo CG Máster, coluna Carbowax). Utilizou-se o hidrogênio (White Martins, $\geq 99,99 \%$ ) como eluente. Os padrões do EMAG foram adquiridos a Sigma-Aldrich.

\section{Resultados e discussão}

Através da extração por solvente das amostras de frutos da palmeira leque-daChina, obteve-se um óleo com aspecto límpido de coloração avermelhada e rendimento aproximado de $10 \%$ em relação a massa de matéria-prima incialmente avaliada. Valores similares foram encontrados por Murillo et al. (2015) que encontraram um rendimento mássico de $9 \%$ para a extração por solvente de amostras de óleo da palmeira leque-daChina.

As análises de índice de acidez do óleo da palmeira leque-da-China tiverem como resultado o teor de $0,9 \%$ de AGL. Knothe et al. (2006) relatam que óleos com teores de índice de acidez superiores a 0,5\% devem ser submetidos ao processo de pré-tratamento dos ácidos graxos livres (AGL) para redução da acidez. Tal processo consiste em uma reação de esterificação por rota ácida, conforme descrito anteriormente na metodologia. Após a etapa de pré-tratamento o conteúdo resultante passa pelo processo de transesterificação alcalina para obtenção do biodiesel.

A Tabela 1 apresenta a composição óleo obtida, bem como de seu respectivo biodiesel em termos dos ésteres metílicos de ácidos graxos (EMAG). Na Figura 3, observase o produto final da reação de transesterificação onde a fase leve é composta por ésteres metílicos de ácidos graxos, excesso de álcool utilizado na reação e traços do catalisador. Essa mistura foi submetida ao processo de purificação visando à obtenção do biodiesel. 


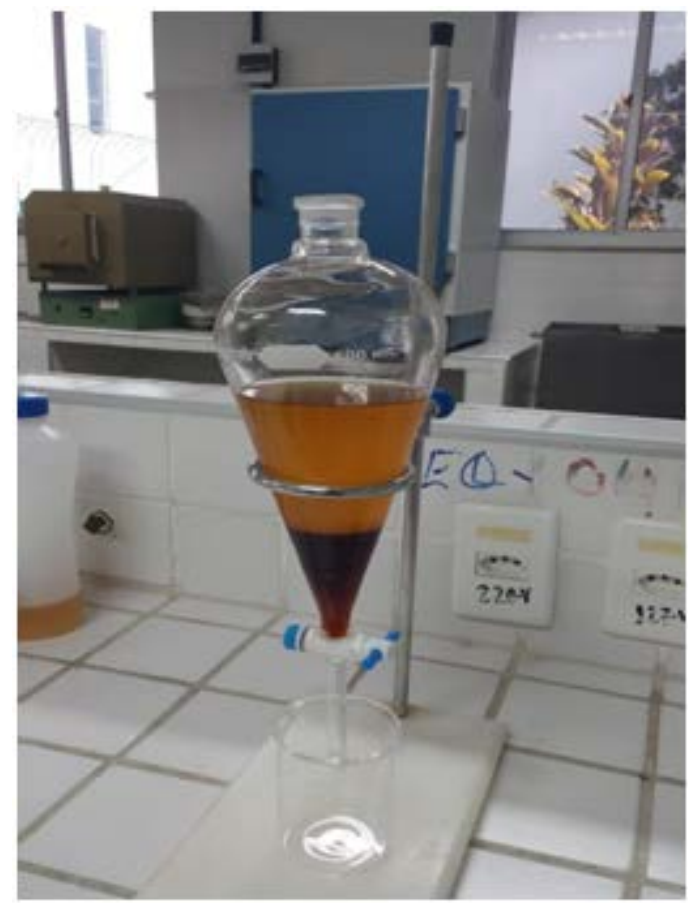

Figura 3. Biodiesel produzido a partir do óleo da palmeira leque-da-China.

Tabela 1. Composição em EMAG do óleo da palmeira leque-da-China e seu biodiesel.

\begin{tabular}{|c|c|c|c|c|c|c|c|c|c|c|}
\hline \multicolumn{11}{|c|}{ Óleo } \\
\hline \multirow{2}{*}{ EMAG } & \multicolumn{4}{|c|}{ Saturado } & \multicolumn{4}{|c|}{ Insaturado } & \multirow{2}{*}{$\begin{array}{l}\text { T.S. } \\
\text { (\%) }\end{array}$} & \multirow{2}{*}{$\begin{array}{l}\text { T.I. } \\
(\%)\end{array}$} \\
\hline & C12:0 & C14:0 & C16:0 & C18:0 & C16:1 & C18:1 & C18:2 & C18:3 & & \\
\hline $\begin{array}{l}\text { Teor } \\
\text { (\%) }\end{array}$ & 3,0 & 1,4 & 52,5 & 2,1 & 3,4 & 34,1 & 3,2 & 0,3 & 59,0 & 41,0 \\
\hline \multicolumn{11}{|c|}{ Biodiesel } \\
\hline \multirow{2}{*}{ EMAG } & \multicolumn{4}{|c|}{ Saturado } & \multicolumn{4}{|c|}{ Insaturado } & \multirow{2}{*}{$\begin{array}{l}\text { T.S. } \\
\text { (\%) }\end{array}$} & \multirow{2}{*}{$\begin{array}{l}\text { T.I. } \\
(\%)\end{array}$} \\
\hline & C12:0 & C14:0 & C16:0 & C18:0 & C16:1 & C18:1 & C18:2 & C18:3 & & \\
\hline $\begin{array}{l}\text { Teor } \\
\text { (\%) }\end{array}$ & 2,9 & 1,3 & 52,8 & 2,8 & 3,1 & 33,7 & 3,4 & 0,1 & 59,8 & 40,2 \\
\hline
\end{tabular}

Ao avaliar os valores da Tabela 1, verifica-se que os teores de ésteres metílicos de ácidos graxos saturados para o biodiesel foi de 59,8\% que segundo Knothe et al. (2006) um biodiesel com tais características (teor de saturados) apresenta maior propensão para cristalização em baixas temperaturas. Como consequência apresentará pontos de névoa e fluidez em temperaturas superiores quando comparados àqueles que apresentam maiores teores de compostos insaturados. Os veículos que utilizam um biocombustível com tais características podem tem problemas de ignição matinal em regiões de temperaturas amenas.

Em contrapartida biodiesel com alto teor de saturados apresentam maior índice de cetano e maior estabilidade a oxidação. Nesse sentido o biocombustível com tais características poderia ser armazenado por mais tempo possuindo assim uma maior aceitação no mercado, desde que seja comercializado em regiões de temperaturas mais altas como o Nordeste brasileiro. 


\section{Conclusão}

Tomando por base os resultados anteriormente apresentados, verifica-se a viabilidade técnica para produção de biodiesel a partir do óleo da palmeira leque-daChina. Devido à alta acidez do óleo o processo de produção do biodiesel foi realizado em duas etapas reacionais o que teoricamente elevaria o custo de produção, por outro lado, esta palmeira apresenta alta produtividade e o óleo proveniente de seus frutos não são aplicados para a indústria alimentícia o que implica em um baixo valor de mercado, sendo assim uma matéria-prima de baixo custo para produção do biodiesel.

Pode-se constatar ainda que a predominância no teor de ésteres saturados presentes no biodiesel favorece uma maior estabilidade oxidativa e índice de cetano, conforme indicado na literatura, tais propriedades são determinantes para um biocombustível de qualidade. Nesse aspecto, os objetivos do presente trabalho foram atingidos ao apresentar uma matéria-prima alternativa para produção de biodiesel, visando a ampliação de possibilidades fontes oleaginosas para produção desse biocombustível.

\section{Conflito de interesses}

Os autores declaram não haver conflito de interesses.

\section{Referências}

ANP - Agência Nacional do Petróleo. Resolução ANP no 798, de 1 de agosto de 2019. Disponível em: <http://legislacao.anp.gov.br/?path=legislacao-anp/resol-anp/2019/ agosto\&item=ranp-798-2019>. Acesso em: 22 dez. 2019.

Costa, C. J.; Marchi, E. C. S. Germinação de sementes de palmeiras com potencial para produção de agroenergia. Planaltina: Embrapa Cerrados, 2008. (Documentos, Embrapa Cerrados; 229). Disponível em: <https://ainfo.cnptia.embrapa.br/digital/bitstream/CPAC2010/30297/1/doc-229.pdf>. Acesso em: 22 dez. 2019.

Knothe, G.; Gerpen, J. V.; Krani, J.; Ramos, L. P. Manual do biodiesel. São Paulo: Edgard Blücher, 2006.

Murillo, R. V.; Leyes, E. A. R.; Canavaciolo, V. L. G.; Perez, R. S.; Delange, D. M.; Sanchez, A. T. L. Composición de ácidos grasos en frutos de Ptychosperma elegans y Livistona chineensis recolectados en Cuba. Revista Cubana de Plantas Medicinales, v. 20, n. 1, p. 142-148, 2015.

Nascimento, F. A.; Cavalcanti, L. A.P. Protótipo de um reator de biodiesel sustentável alimentado energeticamente por energia solar. Revista Brasileira de Energias Renováveis, v. 6, n. 4, p. 748-762, 2017. https://doi.org/10.5380/rber.v6i4.50507

Nwosu, J. N.; Ezegbe, C. C.; Omeire, G. C.; Ahaotu, I.; Owuamanam, C. I.; Udeozor, L. O. Evaluation of the proximate properties of the seed and physicochemical properties of the oil of Chinese fan palm (Livistona chinensis). International Journal of Basic and Applied Sciences, v. 1, n. 4, p. 304-312, 2012. Disponível em: <http://www.crdeepjournal.org/wpcontent/uploads/2012/10/Vol-144-IJBAS2.pdf>. Acesso em: 22 dez. 2019.

Suwanno, S.; Rakkan, T.; Yunu, T.; Paichid, N.; Kimtun, P.; Prasertsan, P.; Sangkharak, K. The production of biodiesel using residual oil from palm oil mill effluent and crude lipase from oil palm fruit as an alternative substrate and catalyst. Fuel, v. 195, p. 82-87, 2017. https://doi.org/10.1016/j.fuel.2017.01.049 
Verma, P.; Sharma, M. P. Review of process parameters for biodiesel production from different feedstocks. Renewable and Sustainable Energy Reviews, v. 62, p. 1063-1071, 2016. https://doi.org/10.1016/j.rser.2016.04.054

CC Informação da Licença: Este é um artigo Open Access distribuído sob os termos da Licença Creative Commons Attribution, que permite uso irrestrito, distribuição e reprodução em qualquer meio, desde que a obra original seja devidamente citada. 